\title{
Dimensions of life definitions
}

Emily C. Parke ${ }^{1,2}$

${ }^{1}$ Philosophy, School of Humanities, University of Auckland, New Zealand

${ }^{2}$ Te Ao Mārama - Centre for Fundamental Inquiry, University of Auckland, New Zealand

PREPRINT - Published (2020) in K. Smith \& C. Mariscal (Eds.) Social and Conceptual Issues in Astrobiology (Oxford University Press)

\section{Life on Mars?}

In early 2018 NASA announced that they had found organic matter on Mars (Potter 2018). Specifically, researchers drilled into Martian rocks and heated them at high temperatures, catalyzing the release of organic molecules trapped there in 3.5-billion-year-old mudslides (Eigenbrode et al. 2018). This finding was presented in major media outlets as an enticing step forward in the search for life on Mars (for example, Chang 2018; Sample 2018).

Organic matter had been found on Mars before, just not in anything near these concentrations (Eigenbrode et al. 2018). Also, these organic molecules are of a sort that could have been produced by life, but could also be produced abiotically, by purely chemical processes. In other words, if life with the biochemistry of Earth life were around, then we would expect to find these sorts of molecules. But finding these sorts of molecules does not mean that life is, or even was, around.

Claims varied about the bearing of this finding on the search for life. Here are some representative examples:

Are there signs of life on Mars? ... We don't know, but these results tell us we are on the right track. (Potter 2018)

Whether it holds a record of ancient life, is the food for extant life, or has existed in the absence of life, organic matter in martian materials holds chemical clues to planetary conditions and processes. (Eigenbrode et al. 2018)

It's not a direct indicator that life may have existed on Mars. (Tamblyn 2018)

This variation is understandable, regarding whether and to what extent this finding points to life. First of all, there are further empirical and theoretical questions to address about the significance of such a finding. Second of all, there is no consensus on what life is in the first place. This chapter will discuss three ways that answers to the question 'What is life?' can vary, and how this variation might bear on life detection efforts in astrobiology.

A natural place to start in thinking about what this recent discovery can tell us about life is the 'NASA definition' of life: "Life is a self-sustaining chemical system capable of Darwinian evolution". This definition was proposed several decades ago by Carl Sagan and popularized in subsequent discussion (Joyce et al. 1994), and is endorsed by NASA in the context of their life detection efforts. ${ }^{1}$

There is no straightforward evidential link between the 2018 finding on Mars and the NASA definition of life. Of course, there is much more to the search for life than this definition. There are

${ }^{1}$ For example at astrobiology.nasa.gov/research/life-detection/about/; accessed March 2019. 
more fine-grained background assumptions at stake which do not figure into this 'official' definition of life at face value: for example, about what life is, what life does, and what life requires. These sorts of assumptions drive the search for organic matter of the sort NASA recently discovered, along with its tentative designation as a small but real mark of success in the search for life on Mars. What this recent finding does is raise the likelihood that there could have been life forms on Mars that are biochemically a lot like life on Earth. That is significant, even if it does not bear much, at face value, on the closest thing to a candidate definition of life at stake here.

This is not just NASA's issue. Anyone looking for life in the universe is in a bit of a tough spot: It cannot be assumed that life elsewhere would resemble life as we know it, in even the most basic ways. So we need some way to recognize it that abstracts away from life as we know it. Many people have thought that coming up with a definition of life is a natural and appealing way to achieve this.

\section{Defining life and why it matters for astrobiology}

Here we run into a puzzle that philosophers and scientists have been arguing about for a long time. In the Western tradition, accounts of the debate about defining life tend to trace it back several millennia to Aristotle, who distinguished living from nonliving things in terms of functions like reproduction and nutrition. Since then, hundreds of others have weighed in, from early modern natural philosophers to contemporary philosophers and scientists. Some have attempted to define life in terms of a single property, like evolution or metabolism; others give a list of properties. Some definitions emphasize biochemical particulars; others are wholly functional. When someone proposes definitional criteria for life, someone else raises a counterexample (for example, if metabolism is sufficient for life, then candle flames arguably qualify as living; if reproduction is necessary for life, then sterile hybrids like mules are problematically disqualified).

Other authors have surveyed extensively the landscape of proposed definitions of life and their various borderline cases and counterexamples (for example, Sagan 1970; Bedau 1998; Luisi 1998; Pályi et al. 2002; Popa 2004; Oliver and Perry 2006; Cleland and Chyba 2007; Bedau and Cleland 2010; Trifonov 2011; Mix 2015; Mariscal and Doolittle 2018). I will not repeat their efforts here. The key points for the purpose of this chapter are that this landscape is substantial (with as many as 100+ definitions of life (Popa 2004; Trifonov 2011)), proposed definitions of life vary widely, and there is no consensus on a definition among astrobiologists, let alone across disciplines with a stake in the matter. ${ }^{2}$

Some have stressed the centrality, fundamentality, or necessity for biology of answering the question 'What is life?' (Cleland and Chyba 2007; Farnsworth et al. 2013; Mix 2015). But while some biologists are interested in that question, biologists in general do not need an answer to it. The subject matter of biology, the living world and its phenomena, is clear enough without one. Borderline cases for defining life do not cause problems for most biologists; for example, the lack of consensus on whether viruses are ultimately nonliving or living does not impede microbiologists' study of bacteria and their viruses.

In contrast to biologists in general, astrobiologists cannot take the status of their subject matter as living or nonliving for granted - its status as such is often precisely what is at stake. There are at least two reasons to think astrobiologists need an understanding of what counts as life. The first is

\footnotetext{
${ }^{2}$ These other disciplines include research on the origin of life, bottom-up synthetic biology, artificial life, and environmental ethics (see discussion in Machery (2012), Bich and Green (2017), Parke (in prep)).
} 
to set search criteria for finding 'life as we don't know it' in the universe. The second is to set success conditions conducive to agreement about when life has been found and when it has not. ${ }^{3}$

In addition to particular cases like the recent Mars finding discussed in Section 1, the meaning of 'life' figures into a broader agenda in astrobiology: looking for biosignatures. There are various ways to spell out what a biosignature is. Here are four sample characterizations:

An observable feature of a planet, such as its atmospheric composition, that our present models cannot reproduce when including the abiotic physical and chemical processes we know about. (Léger et al. 2011; emphasis original)

Distinctive suites of durable textural, mineralogical, or chemical indicators of life. (Campbell 2017)

A feature whose presence or abundance requires a biological origin. (Des Marais et al. 2001)

Evidence that life exists or existed. (Benner 2010)

There is a spectrum here from understandings of biosignatures that require some particular chemical or material assumptions about life, to those that do not. The first two rely on assumptions about biochemistry or the kinds of material traces life leaves; the latter two need not (note that on Benner's characterization of a biosignature, artefacts qualify: If we found technology or AI elsewhere in the solar system, we could be pretty sure that life is or was there to create it (Benner 2010)).

Some understanding of what counts as life is built in to these understandings of what counts as a biosignature. Of course, biosignatures could be conceptualized, and candidate ones assessed, without a definition or even a hypothesis about life beyond life as we know it. We could just take everything we know about life on Earth-its biochemical constraints, its effects on the atmosphere, the microscopic and macroscopic phenomena and artefacts it produces, and so forth - and use that collection of observations to guide the search for biosignatures. Many people invested in finding life beyond Earth want more than that. They want to be in a position to find signatures of life that might be markedly unlike life as we know it, perhaps even at the basic biochemical level.

This desire for grounds to identify 'life as we don't know it' has been a key driver in the search for a definition of life (see discussion in Cleland 2012). Beyond the debate about which definition of life is the definition, in recent years there has also been a meta-debate taking place about the whole project itself. In particular, the proliferation of 100+ proposed candidate definitions of life has led some philosophers and scientists to dismiss the project of trying to define life as pointless or hopeless. This definition skepticism comes in at least three flavors (see discussion in Smith 2018). Carol Cleland has argued that there are too many problems facing the project of strictly defining life, including the sorts of counterexamples and borderline cases discussed above. She says what we need instead is a broader theory of life, but scientists are not in a position to formulate such a theory, because our knowledge of life is based on a single sample: it all descended from the same common ancestor and therefore shares key fundamental properties (Cleland and Chyba 2007; Cleland 2012). Edouard Machery (2012) has argued that scientists use a million different definitions of life, there is no reason to think they will converge on a single, unanimous one, and the project of defining life (as a scientific theoretical concept, anyway) is pointless. Jack Szostak (2012) has argued that science can proceed just fine without worrying about the definition of life, at least in the context of origin of life research.

\footnotetext{
${ }^{3}$ Research on the origin of life is of course another important part of the agenda of astrobiology. For the purpose of this chapter I am focusing in particular on the role of defining life in the search for life in the universe.
} 
In response to these and other pessimists about defining life, several recent discussions have proposed that we should be pluralists about life. That is, we should accept that multiple, even conflicting definitions of life can co-exist, suited to different research agendas. A pluralist position denies that the aim of defining life is to settle on a single definition. In any case, only a subset of the $100+$ definitions of life referred to above are proper definitions in the strict sense that philosophers like to talk about: proposing necessary and sufficient conditions intended for unanimous acceptance. In practice, many so-called definitions of life are less strict. They are better understood as working characterizations of life, which are conceptual frameworks used to guide and make sense of research in a given context, tailored to the agenda of a particular group or field with a stake in understanding life. This is in line with Cleland's (2012) suggestion that astrobiologists search for life with 'tentative criteria', rather than strict definitions, in mind. For further discussion of this idea that definitions of life are often more operational or provisional, and that it is not such bad thing to have more than one of them, see (Oliver and Perry 2006; Griesemer 2015; Mix 2015; Bich and Green 2017).

The combined views of the pessimists and the pluralists point to a grim outlook for settling on a unanimous definition of life, at least today. The positions of pluralism about life, and the more relaxed understanding of what qualifies as a definition, are controversial but popular in the current literature on life-I will not argue for them at length here. For the rest of this chapter I will assume these two positions and ask: How do the ways life is defined bear on scientific practice in astrobiology? In particular, how can the ways life is defined affect how astrobiologists understand search criteria and success conditions for finding life in the universe?

\section{Three ways to define life}

Existing discussions of ways to define life have focused on the content of different definitions, categorizing them into clusters such as evolutionary versus thermodynamic versus metabolic definitions of life. This is often for the purpose of assessing the relative merits and implications of different categories, and endorsing one category as fundamental (Sagan 1970; Pályi et al. 2004; Popa 2004; Kompanichenko 2008; Trifonov 2011; Mix 2015). Below I discuss something different: three ways to categorize definitions that vary in their strategies or commitments regarding how life is defined. These abstract away from the specific content or focal feature(s) of definitions of life. There are at least three such dimensions along which definitions of life can vary: Treating living/nonliving as a dichotomy or a matter of degree, defining living individuals or living collectives, and defining life materially or functionally. ${ }^{4}$ I discuss each in turn and suggest how variation in each dimension can affect the role of the concept of life in setting search criteria or success conditions in astrobiology.

\subsection{Living/nonliving: Dichotomy or matter of degree?}

Most proposed definitions of life offer a way to distinguish between two categories, living and nonliving. In particular, they specify criteria for drawing a line that will include what is living and exclude what is nonliving. A paradigm example of this is Maturana and Varela's "All that is living must be based on autopoiesis, and if a system is discovered to be autopoietic, that system is defined as living, i.e., it must correspond to the definition of minimal life" (1973, cited in Popa 2004). An alternative is to treat the difference between living and nonliving explicitly as a continuum, or a matter of degree. Several recent accounts have done this: they specify a few features as definitional of life, but life comes in degrees. Christophe Malaterre's (2010) account specifies five features

\footnotetext{
${ }^{4}$ These are three important ones; I think there are others as well. For example, other relevant dimensions include (1) pragmatic versus theoretical definitions of life, and (2) whether life is treated as a (natural) kind, as is the norm, or not (for arguments that life is not a natural kind see Hermida 2017; Mariscal and Doolittle 2018).
} 
(individuation, replication, variation, metabolism, and coupling of components) and allows systems to instantiate each of those features to varying degrees. Mark Bedau's (2012) account specifies three features (a container, program, and metabolism) and allows for a spectrum of Boolean combinations of those features and their relationships of mutual support and integration, shading from nonliving to living. On these matter-of-degree accounts, there is no matter of fact about a clear line between what is nonliving and living, just a gradual scale.

Treating nonliving/living as a dichotomy versus a matter of degree will make a difference to the sorts of claims that can be made about life in the universe. Much of the debate about life has treated the matter of defining life as if the aim is to demarcate two kinds or categories, living and nonliving. A clean divide between what is nonliving and what is living might seem naturally appealing in the context of finding life in the universe. It would be nice to have unambiguous yes-or-no answers when candidate life forms are identified. It would be nice - once the empirical details are sorted out, which is of course no small matter- to be able to say in a given case that life-detection missions like NASA's had either found life, or had not.

On the other hand, understanding life as a matter of degree better reflects the truth of the matter in thinking about the origin of life, on Earth or anywhere. It makes sense for the search for life on other planets to understand life in a way that explicitly captures the possibility of discovering not only living or nonliving things, but also intermediaries: minimal or marginal cases of life, where life is understood as something that comes in degrees. Astrobiologists are not looking only for living organisms or populations. They are looking for evidence that life could have once existed elsewhere in the universe, or could be in the process of emerging elsewhere. To the extent that the latter constitutes part of the agenda of searching for life, it makes sense to conceptualize life as a matter of degree.

If living/nonliving is understood as a matter of degree rather than two dichotomous categories, the possibility space for interpreting life-detection findings, like the ones discussed at the outset of this chapter, can be understood differently. Discussions have tended to frame that possibility space in terms of three options: life was found, life was not found, or the results are ambiguous between biotic and abiotic explanations. A fourth option - which is consistent with the third option, but backgrounded in discussions treating living/nonliving as a dichotomy-could be that there are systems on Mars (for example) best understood as transitional between paradigm cases of nonlife and paradigm cases of life, as one would have found on Earth sometime in the window between roughly 4.3 and 3.8 billion years ago. A clearer shared understanding of the in-between status of such not-fully-living (but not nonliving) forms would be an ideal starting point for detecting them.

\subsection{Living individuals or collectives?}

Many existing definitions of life propose grounds to separate living organisms, like lizards and lactobacilli, from nonliving things like liposomes and laptops. This is a natural way to understand the question 'What is life?' - in terms of what makes living individuals living. Some definitions of life, however, are based on properties that by definition apply only to collectives of individuals, or collectives of individuals over time. An example of the former sort of property is variation; an example of the latter sort is evolution. These appear in many popular definitions of life, including the NASA definition discussed in Section 1. When variation or evolution are cited as definitional features of life, the bearer of those features is a population, not an individual. So while the question is often interpreted as asking what distinguishes a living thing (organism) from a nonliving thing, a number of authors treat living populations (for example, Bedau 1998, Smith 2018) or even the biosphere (Lovelock 1979, Feinberg and Shapiro 1980) as the focal unit in characterizing life, rather than individual organisms. 
There is no principled reason to prefer defining living individuals to defining living collectives, nor is there any principled problem with including features of both levels of biological organization in the same definition of life. Plenty of definitions do this. But there are some potential conceptual complications with this, in the context of searching for life in the universe.

The features emphasized in definitions of life are not always the same as the features targeted by life-detection tools and techniques. Furthermore, they are often framed at a different level (that of individual organisms versus collectives of them). Biologists and astrobiologists use different tools and timescales to look for evidence of evolutionary processes in nature, as opposed to evidence of living things, like microbes. Regarding tools, the 1976 Mars Viking missions involved techniques by which researchers could in principle identify traces of microbes and their metabolic activity; the same is true for bids for sites for the upcoming 2020 Rover missions. These approaches to finding life in the universe prioritize looking for evidence of microbial life forms, not (direct) evidence of evolution or variation. There are other ways to look for evolution and variation. With our familiar microbial Earth life, this is typically done by looking for genetic markers (ribosomal RNA sequences) and their change over time, or their differences with respect to one another or to a putative common ancestor. It is not obvious how these methods would translate to looking for life as we don't know it, given their reliance on particulars not only of DNA-based biochemistry but of conserved genome regions common to familiar life.

Regarding timescales, if life is understood purely in terms of features of individual organisms and we are searching for one, we could in principle find one (or evidence of one) in an instant. Finding evolutionary processes, or direct evidence of them, has to be done over time (but see Benner 2010 for a suggested way around this, discussed in Section 3 below). So variation in this feature of how life is defined could make an empirical and theoretical difference. Specifying criteria for finding living individuals, versus living evolving populations, can influence the kinds of signatures of life astrobiologists look for, the tools they use to look for them, and the timescales on which they can do so. Of course, the projects of searching for living individuals and evolving populations are compatible, and can be pursued concurrently. But they can be conceptually and practically distinguished in ways that matter for astrobiology.

\subsection{Material or functional?}

Some definitions of life specify material particulars like biochemistry, carbon-based biochemistry, or nucleic acids. For example, Perrett (1952) defines life as "a potentially self-perpetuating system of linked organic reactions, catalyzed stepwise and almost isothermally by complex and specific organic catalysts which are themselves produced by the system." Other definitions are purely functional, and invite a variety of material or even digital instantiations of life as we don't know it. An example is "life is self-reproduction with variation" (Trifonov 2011), which could be instantiated by familiar carbon-based life, silicon-based life, self-replicating computer programs, and any number of other systems, chemical or otherwise. How material or functional a definition is can come in degrees: for example, Perrett's 'linked organic reactions' specifies a more fine-grained material feature than the NASA definition's criterion that life is a 'chemical system'. Furthermore, many definitions of life combine material and functional elements.

Purely functional definitions leave more room for finding life in the universe that does not resemble our current sample of life in even its most basic biochemical aspects. On the other hand, they give less guidance about what to look for. Many definitions of life combine functional with loosely material elements - they are based on a coarse-grained material understanding of life with reference to chemistry, but without more fine-grained assumptions about molecular specifics like nucleic 
acids. These include, for example, the NASA definition, and Pace's (2011) definition of life as "a self-replicating, evolving system expected to be based on organic chemistry".

The more material details a definition of life specifies, the more readily it allows for direct specific claims about biosignatures: what specifically to search for, or whether a particular finding qualifies as life or a sign of life. Purely functional definitions will not do this directly. But they can provide a template for more specific guidance on searching for life, once the appropriate details are filled in. Benner (2010) exemplifies this sort of reasoning, connecting a relatively functional definition of life to more specific claims about biochemical signatures of life. He takes the NASA definition ("life is a self-sustaining chemical system capable of Darwinian evolution") as a starting point, and supplements it with the assumption that chemical systems capable of Darwinian evolution must be based on polyelectrolites (molecules with repeating charges in their backbone). He argues that we should focus the search for life on polyelectrolites, and evidence of polyelectrolites would qualify as evidence of life under that definition.

\section{Conclusion}

I have discussed three dimensions on which accounts of life can vary, and suggested that variation along each of these dimensions can affect how the concept of life figures in to theoretical and empirical efforts in astrobiology: in forming search criteria for life in the universe, understanding success conditions, and communicating about them. In summary:

- Living/nonliving as a dichotomy or a matter of degree: Dichotomous understandings of living/nonliving might seem conducive to clearer answers about the status of discoveries in the universe; matter-of-degree understandings are conducive to clearly recognizing transitional entities as such.

- Living individuals or collectives: Focusing on features of individual life forms (such as selfreproduction and metabolism), versus features of collectives of individuals (such as variation and evolution), can influence both conceptual search criteria for life and the tools and techniques involved.

- Material versus functional understandings of life: More material understandings of life give clearer guidance without supplementation with auxiliary assumptions. More functional understandings leave more room for interpretation and enable searching for 'life as we don't know it' at even the most basic chemical level.

The second and third dimensions, especially, are not straightforward either/or choices. For example, some definitions of life specify only properties of individuals, some specify only properties of populations, and others explicitly address both (for example, Ruiz-Mirazo et al. 2004). Definitions can be specifically material, permissively functional, or in between. So these three dimensions do not give us a simple possibility space of eight ways to define life. Rather, we can think of them as axes along which approaches to defining life can be assessed or compared.

The aim of this short chapter is not to make value judgments about the superiority of any particular way to define of life, along any of these three dimensions - at least not in a wholesale way across astrobiology, let alone across fields with a stake in the concept of life (see footnote 2). Rather, this is a call for clearer communication about which understanding of life is at stake in the context of a given project or finding. And, specifically, how that understanding of life relates to search criteria and success conditions for finding life or signs of life. Explicitly recognizing a plurality of ways to define life in the sense discussed here (in addition to the range of focal features regarded as 
definitional of life) is a starting point for clearer discussion of the background assumptions at stake in life-detection efforts.

\section{Acknowledgments}

I am grateful to Kathy Campbell, Carlos Mariscal, Kelly Smith and an anonymous reviewer for helpful feedback on earlier drafts.

\section{References}

Bedau M (1998) Four puzzles about life. Artificial Life, 4(2), 125-140.

Bedau M (2012). A functional account of degrees of minimal chemical life. Synthese, 185(1), 7388.

Bedau M \& Cleland CE (Eds.) (2010) The nature of life: Classical and contemporary perspectives from philosophy and science. Cambridge University Press.

Benner SA (2010). Defining life. Astrobiology, 10, 1021-1030.

Bich L \& Green S (2017). Is defining life pointless? Operational definitions at the frontiers of biology. Synthese, published online 18 April 2017, 1-28.

Campbell C (2017) Hot springs and earliest life on land. Posted on A History of Stones, 2

November, www.stonehistorian.space/2017/11/hot-springs-and-earliest-life-on-land.html (accessed March 2019).

Chang K (2018) Life on Mars? Rover's latest discovery puts it 'on the table'. The New York Times, 7 June 2018. Available at www.nytimes.com/2018/06/07/science/mars-nasa-life.html (accessed November 2018).

Cleland CE (2012). Life without definitions. Synthese, (185), 125-144.

Cleland CE \& Chyba CF (2007) Does "life" have a definition? In W. T. Sullivan \& J. A. Baross (Eds.), Planets and life: The emerging science of astrobiology. Cambridge University Press.

Des Marais DJ, Harwit MO, Jucks KW, Kasting JF, Lin DN, Lunine JI, ... \& Woolf NJ (2002). Remote sensing of planetary properties and biosignatures on extrasolar terrestrial planets. Astrobiology, 2(2), 153-181.

Eigenbrode JL, Summons RE, Steele A, Frissinet C, Millan M, Navarro-Gonzalez R, Sutter B, MAdam AC, Franz HB, Glavin DP, Archer PD Jr, Mahaffy PR, Conrad PG, Hurowitz JA, Grotzinger JP, Gupta S, Ming DW Sumner DY, Szopa C, Malespin C, Buch A, \& Coll P (2018) Organic matter preserved in 3-billion-year-old mudstones at Gale crater, Mars. Science 360(6393): 1096-1101.

Farnsworth KD, Nelson J \& Gershenson C (2013). Living is information processing: From molecules to global systems. Acta Biotheoretica, 61(2), 203-222. 
Feinberg G \& Shapiro R (1980). Life beyond Earth: The intelligent earthling's guide to life in the universe. William Morrow.

Griesemer J (2015). The enduring value of Gánti's chemoton model and life criteria: Heuristic pursuit of exact theoretical biology. Journal of Theoretical Biology, 381, 23-28.

Hermida M (2016). Life on Earth is an individual. Theory in Biosciences 135: 37-44.

Joyce GF, Deamer DW \& Fleischaker G (1994) Origins of life: the central concepts, forward. Jones and Bartlett.

Kompanichenko V (2008). Three stages of the origin of life process: bifurcation, stabilization and inversion, International Journal of Astrobiology, 7(1), 27-46.

Léger A, Fontecave M, Labeyrie A, Samuel B, Demangeon O \& Valencia D (2011) Is the presence of oxygen on an exoplanet a reliable biosignature? Astrobiology, 11(4), 335-41..

Lovelock J (1979). Gaia: A new look at life on Earth. Oxford University Press.

Luisi PL (1998) About various definitions of life. Origins of Life and Evolution of the Biosphere, $28,613-622$.

Machery E (2012) Why I stopped worrying about the definition of life... and why you should as well. Synthese, 185(1), 145-164.

Malaterre C (2010). Lifeness signatures and the roots of the tree of life. Biology and Philosophy, 25, 643-658.

Mariscal C \& Doolittle F (2018). Life and Life only: A radical alternative to life definitionism. Synthese, https://doi.org/10.1007/s11229-018-1852-2.

Mix LJ (2015) Defending definitions of life. Astrobiology, 15(1), 15-19.

Oliver JD \& Perry RS (2006) Definitely life but not definitively. Origins of Life and Evolution of Biospheres, 36(5-6), 515-521.

Pace NR (2001). The universal nature of biochemistry. Proceedings of the National Academy of Sciences of the United States of America, 98(3), 805.

Pályi G, Zucchi C \& Caglioti L (Eds.) (2002) Fundamentals of life. Paris: Elsevier.

Parke EC (in preparation). Finding, explaining, and engineering life.

Perret M (1952). Biochemistry and bacteria. New Biology, 12, 68.

Popa R (2004) Between necessity and probability: Searching for the definition and origin of life. Springer.

Potter S (2018) NASA finds ancient organic material, mysterious methane on Mars. NASA Press release. Available at www.nasa.gov/press-release/nasa-finds-ancient-organic-material-mysteriousmethane-on-mars (accessed November 2018).

Ruiz-Mirazo K, Peretó J \& Moreno A (2004) A universal definition of life: Autonomy and openended evolution. Origins of Life and Evolution of the Biosphere, 34, 323-46. 
Sample I (2018) NASA Mars rover finds organic matter in ancient lake bed. The Guardian, 7 June 2018. Available at www.theguardian.com/science/2018/jun/07/nasa-mars-rover-finds-organicmatter-in-ancient-lake-bed (accessed November 2018).

Sagan C (1970) Life. Encyclopedia Britannica (pp. 1083-1083A). Chicago: Encyclopaedia Britannica Incorporated.

Smith K (2018). Life as adaptive capacity: Bringing new life to an old debate. Biological Theory, 13(2), 76-92.

Szostak JW (2012). Attempts to define life do not help to understand the origin of life. Journal of Biomolecular Structure and Dynamics, 29(4), 599-600.

Tamblyn T (2018) NASA's Mars rover has found organic matter on the planet's surface. Huffington Post, 8 June 2018. Available at www.huffingtonpost.co.uk/entry/nasas-mars-rover-has-foundorganic-matter-on-the-planets-surface_uk_5b1a4e2ce4b0bbb7a0db7486 (accessed November 2018)

Trifonov EN (2011) Vocabulary of definitions of life suggests a definition. Journal of Biomolecular Structure and Dynamics, 29(2), 259-266. 